\title{
Metallointercalator [Ru(dppz)2(PIP)]2+ renders BRCA wild-type triple-negative breast cancer cells hypersensitive to PARP inhibition
}

\begin{abstract}
There is a need to improve and extend the use of clinically approved poly(ADP-ribose) polymerase (PARP) inhibitors (PARPi), including for BRCA wild-type triple-negative breast cancer (TNBC). The demonstration that ruthenium(II) polypyridyl complex (RPC) metallointercalators can rapidly stall DNA replication fork progression provides the rationale for their combination alongside DNA damage response (DDR) inhibitors to achieve synergism in cancer cells. The aim of the present study was to evaluate use of the multi-intercalator $[\mathrm{Ru}(\mathrm{dppz}) 2(\mathrm{PIP})] 2+(\mathrm{dppz}=\operatorname{dipyrido}[3,2-\mathrm{a}: 2$ ',3'-c]phenazine, PIP $=(2-($ phenyl $)$ imidazo[4,5f][1,10]phenanthroline, Ru-PIP) alongside the PARPi olaparib and NU1025. Cell proliferation and clonogenic survival assays indicated a synergistic relationship between Ru-PIP and olaparib in MDA-MB-231 TNBC and MCF7 human breast cancer cells. Strikingly, low dose Ru-PIP renders both cell lines hypersensitive to olaparib, with a >300-fold increase in olaparib potency in TNBC, the largest nongenetic PARPi enhancement effect described to date. A negligible impact on the viability of normal human fibroblasts was observed for any combination tested. Increased levels of DNA double-strand break (DSB) damage and olaparib abrogation of Ru-PIP-activated pChk1 signaling are consistent with PARPi-facilitated collapse of Ru-PIP-associated stalled replication forks. This results in enhanced G2/M cell-cycle arrest, apoptosis, and decreased cell motility for the combination treatment compared to single-agent conditions. This work establishes that an RPC metallointercalator can be combined with PARPi for potent synergy in BRCA-proficient breast cancer cells, including TNBC.
\end{abstract}

